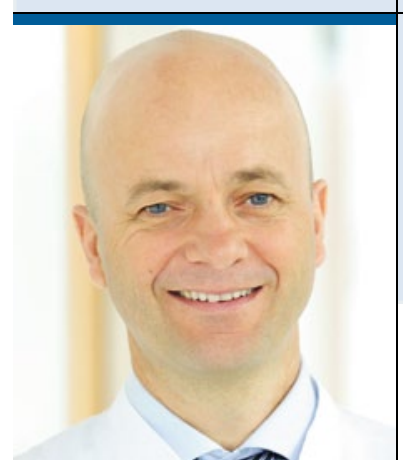

Prof. Dr. G. Nickenig

Medizinische Klinik u. Poliklinik II

Universitätsklinikum Bonn

\title{
Grundlagenforschung, Risikomanagement und Therapie der KHK
}

W enn auch die strukturellen Herzerkrankungen durch zahlreiche Innovationen ganz im Mittelpunkt des Öffentlichkeitsinteresses stehen, so ist und bleibt die koronare Herzerkrankung die Herausforderung schlechthin in der kardiovaskulären Medizin und vielleicht auch in der Medizin insgesamt, wenn man sich Morbiditäts- und Mortalitätsraten vergegenwärtigt.

Die Grundlagenforschung im Bereich der Atherosklerose gehört zu dem Spannendsten, was die Herzmedizin zu liefern hat; hier sind in den letzten Jahren grundsätzlich neue Erkenntnisse, insbesondere im Bereich der Inflammation, Immunologie und Lipide gewonnen worden, die in nicht allzu ferner Zukunft auch unseren

"Die KHK ist die Herausforderung nicht nur der kardiovaskulären, sondern der Medizin insgesamt."

Nach und neben diesen Lebensstilveränderungen sind medikamentöse Therapieprinzipien der koronaren Herzerkrankung von allergrößter Bedeutung in der täglichen Praxis. Auch hier lohnt sich der Blick über den Tellerrand; welche Medikamente werden tatsächlich und darüber hinaus auch noch langfristig benötigt?

Die Innovationspalette in der interventionellen Behandlung der koronaren Herzerkrankung ist groß, und hier gibt es zahlreiche neue Dinge zu berichten, die vielleicht dann auch die Therapie der koronaren Dreigefäßerkrankung beeinflussen könnten eine der wesentlichen Kontroversen in der heutigen Herzmedizin, Bypassoperation oder Intervention?

Ich bin sicher, die Autoren dieses Heftes haben die richtige Mischung klinischen Alltag verändern sollten. Solange die spezifischen Grundlagen der Atherosklerose jedoch nicht ausreichend geklärt sind und sich daraus entwickelnde spezifische therapeutische Maßnahmen noch nicht zur Verfügung stehen, bildet das Risikofaktormanagement die wesentliche Basis der Behandlung der KHK. Hier muss immer wieder hinterfragt werden, was tatsächlich evidenzbasiert ist und welche Maßnahmen wir unseren Patienten unnachgiebig nahebringen müssen.

Ihr

Prof. Dr. Georg Nickenig zwischen Hintergrundinformation und praktischen Hinweisen getroffen, und ich wünsche Ihnen viel Freude bei der Lektüre. Patienten untachgiebig nahebringen müssen. de bei der Lektüre.

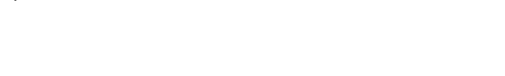

